\title{
A Huge True Axillobrachial Aneurysm Following Arteriovenous Fistula Ligation
}

\section{(1) Orhan Atilla1, ๑ Ufuk Özergin²}

${ }^{1}$ Selçuk University, Faculty of Medicine, Department of Cardiovascular Surgery, Konya, Turkey

${ }^{2}$ Konya Farabi Hospital, Clinic of Cardiovascular Surgery, Konya, Turkey

\begin{abstract}
Aneurysmal degeneration of the upper limb arteries is a rare entity. We discuss the clinical characteristics, surgical options for a huge true axillobrachial artery aneurysm following arteriovenous fistula closure after renal transplantation. The underlying pathogenesis of the true axillobrachial artery aneurysm remains unclear. The current theory is that the adaptive arterial wall enlargement
\end{abstract}

\section{Introduction}

Aneurysmal degeneration of the upper limb arteries is a rare entity (1). A true aneurysm formation that is proximal to the inflow artery after closing an arteriovenous fistula (AVF) is a rare complication (2), and the underlying pathogenesis of a true axillobrachial artery aneurysm (TABA) remains unclear (3). The is secondary to the increased flow in the inflow artery. Immunosuppressive therapy and corticosteroids contribute to the aneurysm formation. Surgical treatment strategies including aneurysmal excision, bypass with autogenous or prosthetic grafts are the primary therapeutic options.

We believe that this case is especially helpful for young surgeons.

current theory is that the adaptive arterial wall enlargement is secondary to the increased flow in the inflow artery (3), and immunosuppressive therapy and corticosteroids contribute to aneurysm formation (2). Surgical treatment, including excision and bypass, is the primary therapeutic option (1). The surgeons must be careful to identify patients with a TABA in order to provide the required surgery in time. 
This paper explains our surgical approach to the treatment of a TABA that occurs after AVF ligation following successful renal transplantation (RTx).

\section{Case report}

A 63-year-old female with complaints of increasing pain, swelling, and paresthesia in her left arm was admitted to the clinic. The patient had a 30-year history of chronic renal failure, and the left brachiocephalic AVF was used for hemodialysis without any revision. She underwent successful RTx in 2008. The AVF was ligated at the level of the anastomosis with a single surgical tie in 2009. No additional information was available regarding the procedure because the operation was performed at another hospital. For this reason, there is no information about the diameter of the brachial artery or whether or not duplex ultrasonography (DUS) was performed during ligation. She received immunosuppressive therapy for nine years. During the long postoperative period, the swelling in the left arm of the patient expanded gradually. The patient was admitted to several hospitals, but she did not receive any treatment because of the potential surgical risks.

On physical examination, paresthesia, muscle weakness, and a huge pulsatile mass were detected in the left upper limb (Figure 1A). There were ischemic changes and weakened distal pulses in her left hand.

The DUS showed a true aneurysm of $7 \mathrm{~cm}$ in diameter originating from the left axillobrachial artery. The aneurysm extended to the brachial artery bifurcation and was highly tortuous. The aneurysm examination was performed using DUS because CT angiography (CTA) was not available.

Because of the excessive dilatation of the aneurysm, surgical repair of the aneurysm was offered under elective conditions.

In the operation, a left subclavicular incision was made, and the left subclavian artery was controlled. A longitudinal incision was made in the aneurysm, the aneurysmal mass was exposed (Figure 1B), and the distal arteries were clamped. A saphenous vein graft was harvested. The aneurysm was extended from the axilla to the forearm. We preferred a composite graft in order to prevent the potential risk of a kink in the prosthetic graft at the elbow level and to avoid diameter incompatibility in the axillary region. The composite graft interposition was performed before the aneurysmal sac resection to avoid a long ischemia time (Figure 2A). The total arterial occlusion time was 26 min. The median nerve was carefully dissected from the aneurysmal wall. After the aneurysm was removed, the reversed vein graft was resized to fit its anatomical trace. Finally, the enlarged skin flap was removed. The operation was completed without complications. The postoperative follow-up was uneventful (Figure 2B). At two years follow-up, there were no ischemic or neurologic events in the left upper extremity and the graft was patent.

Written informed consent was obtained from the patient for publication of this case report and accompanying images. A copy of this consent is available upon request.
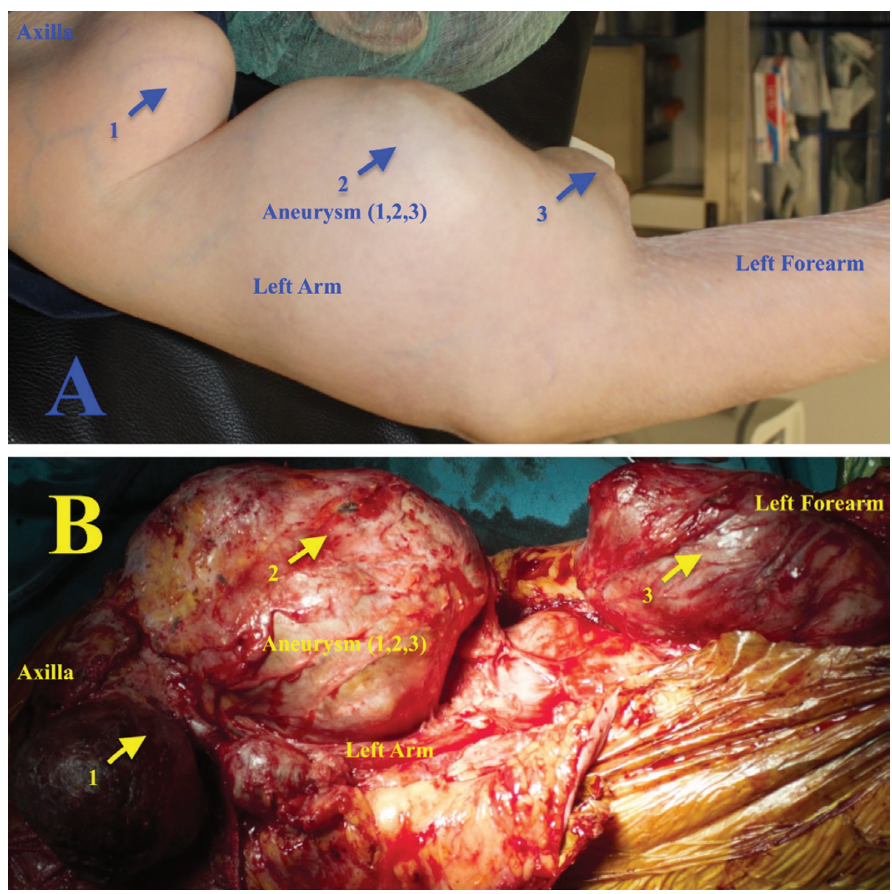

Figure 1. Preoperative view of the left arm (A) and intraoperative view of the aneurysmal exploration (B). 

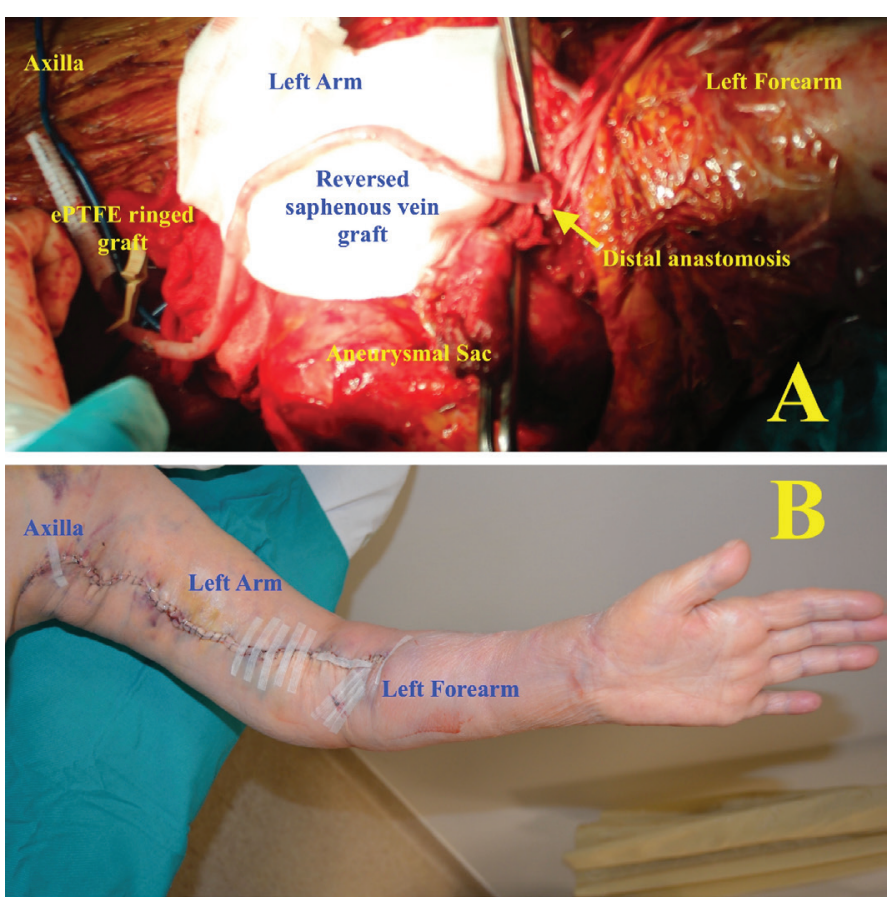

Figure 2. The revascularization procedure: (A) and postoperative day\#3 view of the left arm (B).

\section{Discussion}

Aneurysm formation is a serious complication of vascular access. The surgical approach for treating a huge TABA requires the use of a combination of experiences in most cases. The decision to attempt a surgical reconstruction in the case of a huge TABA can be a nightmare for a surgeon due to the possible complications (4).

Axillobrachial aneurysms are rare entities and may occur for many reasons, including multiple vascular traumas, vasculitis, and endocarditis (5). A TABA is a very rare condition (4). It is difficult to detect progression in a TABA because of its unknown nature. Duplex imaging is a simple method for following the enlargement of the brachial artery over time (3). However, we could not get sufficient information about the previous imaging results and follow-up because the patient underwent follow-up in another hospital. Although other true peripheral aneurysms are seen as synchronous with each other at certain times, there is no relation between TABAs and other peripheral aneurysms (1). This suggests that TABAs have a different pathogenesis.

Many cases in the literature suggest a relation between TABAs and AVFs (3-5). While some studies correlate this relationship to AVF creation (3), some also correlate it to AVF closure $(4,5)$. Currently, the pathogenesis is explained by the fact that the shear forces arising from the increased flow in the arterial lumen cause the dilation of the arterial wall (3). Even if the AVF is completely closed, this process cannot be stopped, and a permanent arteriomegaly will develop (6). According to Eugster's results, the aneurysm formation in the brachial artery following AVF creation is time-dependent. He also argues that high flow through the AVF is the main trigger for aneurysm formation (6). We cannot explain the pathophysiological process from a single patient, and we cannot provide a histopathological sample for the aneurysm because of the inadequate infrastructure. As in our case, RTx and immunosuppressive drugs may be related to the progression of a true aneurysm (2). Investigations have shown that TABAs are more commonly observed in transplant patients than in non-transplant patients (6). The use of post-transplant immunosuppressive agents and corticosteroids also contributes to the progression of the aneurysm (2).

TABAs have clinical findings similar to other peripheral aneurysms. Most of the cases are asymptomatic. Clinical manifestations include pain, ischemic changes, and neurological findings due to local compression (5). In our case, there was pain, ischemia, paresthesia, and muscle weakness.

The physical examination should include both neurological examination and vascular examination (5). DUS is the first step in diagnosis and is highly reliable (5). CTA may be used in selected cases, particularly when planning an operation (5). We performed the preoperative examinations using DUS because CTA was not available. However, we believe that DUS is sufficient for pre- and post-operative follow-up.

The main option in TABA treatment is surgery $(2,4$, $5)$. In very rare cases, such as with vasculitis, medical 
treatment may be tried as the first management (1). However, if the aneurysm is large, surgery is the primary option (4). In most studies, surgery is recommended when the artery diameter is 1.5 to 2 times larger than normal (1). We support this view, but most surgeons are reluctant to operate on huge aneurysms (1). Our patient required the operation very early, but she did not accept because of its high risk. Surgical interventions usually include excision, ligation, and bypass grafting. Venous patches may also be used in anatomically suitable cases (1-6).

Because of the huge size of the aneurysm, we performed a different surgical technique, as detailed above. Because the subclavian artery was dilated and the aneurysm extended under the elbow, there was a graft mismatch risk. For this reason, we preferred to use a composite graft. In similar cases, the saphenous vein has been used as a conduit (1-6). Prosthetic grafts or other native veins have been used in a few cases (4).

There are also new techniques in the literature, especially about preservation of the native AVF. When the preservation is not the concern, especially for huge AVF aneurysms, Tan-Gor technique should also be evaluated for excessive skin tissue, which dramatically reduces operational time (7).

Currently, endovascular interventions are feasible with suitable true aneurysms and pseudoaneurysms associated with vascular-access procedures, but they are not convenient with huge TABAs $(1,4)$.

\section{Conclusion}

We believe that proximal arterial control is useful for reducing the risk of bleeding in cases of huge aneurysms and those complications can be minimized using an autogenous and non-autogenous graft combination in difficult cases.

\section{Ethics}

Informed Consent: Written informed consent was obtained from the patient.

Conflict of interest: No conflict of interest declared by the authors.

Financial Disclousure: The authors declared that this study has no financial support.

\section{References}

1. Wong SSN, Roche-Nagle G. Giant True Brachial Artery Aneurysm. Vascular and Endovascular Surgery. 2012;46(6):492-4.

2. Teixeira S, Pinto PS, Veiga C, Silva I, Almeida R. Aneurysmal Degeneration of the Brachial Artery after Vascular Access Creation: Surgical Treatment Results. Int J Angiol. 2017;26(3):186-90.

3. Mestres G, Fontsere N, Yugueros X, Tarazona M, Ortiz I, Riambau V. Aneurysmal degeneration of the inflow artery after arteriovenous access for hemodialysis. Eur J Vasc Endovasc Surg. 2014;48(5):592-6.

4. Marzelle J, Gashi V, Nguyen HD, Mouton A, Becquemin JP, Bourquelot P. Aneurysmal degeneration of the donor artery after vascular access. J Vasc Surg. 2012;55(4):1052-7.

5. Cleveland EC, Sinno S, Sheth S, Sharma S, Mussa FF. Neurovascular compromise due to true brachial artery aneurysm at the site of a previously ligated arteriovenous fistula: Case report and review of literature. Vascular. 2015;23(6):668-72.

6. Eugster T, Wigger P, Bolter S, Bock A, Hodel K, Stierli P. Brachial artery dilatation after arteriovenous fistulae in patients after renal transplantation: a 10-year follow-up with ultrasound scan. J Vasc Surg. 2003;37(3):564-7.

7. Tanyeli Ö, Görmüş N. Treatment of arteriovenous fistula aneurysms in hemodialysis patients and introduction of Tan-Gor skin flap technique. Damar Cer Derg 2018; 27(1): 008-016. 